\title{
Roles of GFPT2 Expression Levels On the Prognosis and Tumor Microenvironment of Colon Cancer
}

\section{Xiaorong Ding}

The Affiliated Huaian No.1 People's Hospital of Nanjing Medical University

Hua Liu

The Affiliated Huaian No.1 People's Hospital of Nanjing Medical University

\section{Ying Yuan}

The Affiliated Huaian No.1 People's Hospital of Nanjing Medical University

\section{Qin Zhong}

The Affiliated Huaian No.1 People's Hospital of Nanjing Medical University

Xiaomin Zhong ( 13912074971@163.com )

The Affiliated Huaian No.1 People's Hospital of Nanjing Medical University

\section{Research Article}

Keywords: colon cancer, GFPT2, prognosis, tumor microenvironment (TEM)

Posted Date: September 30th, 2021

DOI: https://doi.org/10.21203/rs.3.rs-923669/v1

License: (c) (i) This work is licensed under a Creative Commons Attribution 4.0 International License.

Read Full License

Version of Record: A version of this preprint was published at Frontiers in Oncology on March 7th, 2022. See the published version at https://doi.org/10.3389/fonc.2022.811559. 


\section{Abstract}

Background Recently, increasing evidence has suggested that Glutamine-fructose-6-phosphate transaminase 2 (GFPT2) is related to carcinogenesis. However, the potential roles of GFPT2 in colon cancer still need to be fully investigated.

Methods We examined the protein levels of GFPT2 by immunohistochemistry (IHC) in tissues collected from 83 patients with colon cancer. We checked the relationship between GFPT2 expression levels and overall survival (OS), stromal and immune scores and immune components from The Cancer Gene Atlas (TCGA) database. Expression of GFPT2 in single cell subpopulations was calculated from The Tumor Immune Single Cell Center (TISCH). GFPT2 levels and drug sensitivity data were performed from CellMiner dataset.

Results GFPT2 was highly expressed and correlates with poor pathological features in 83 colon cancer patients. Moreover, increased GFPT2 expression was significantly associated with poorer OS in 329 colon adenocarcinoma (COAD). Gene oncology (GO) and KEGG analysis showed the differentially expressed genes of GFPT2 were mostly enriched in focal adhesion, ECM receptor interaction, JAK/STAT signaling pathway and immune related pathways. In addition, GFPT2 levels were correlated with the tumor microenvironment (TEM). GFPT2 expression was linked to CAFs-associated factors and EMT-related factors. GFPT2 was positively correlated with immunosuppressive cells and regulated immunosuppressive factors and T-cell exhaustion. Finally, our data suggested that the expression of GFPT2 may be a judgment of the sensitivity of a certain class of drugs.

Conclusions Our work revealed the roles of GFTP2 in tumorigenesis, especially in immune response, tumor microenvironment and drug resistance, which is crucial for the development of customized cancer therapies.

\section{Background}

Colon cancer is a common malignant tumor of the gastrointestinal tract occurring at the junction of the rectum and sigmoid colon, with the highest incidence in the age group of 40 to 50 years old, and the ratio of men to women is 2 to $3: 1$ (1). Globally, colon cancer is the third and second most commonly diagnosed cancer in men and women, respectively, with 1.9 million new cases and nearly 935,000 deaths in 2020 (2). Incidence and mortality rates are significantly higher in men than in women. Colon cancer tumor cells usually invade the lymphatic vessels, blood vessels or other channels from the primary site and continue to grow there, forming the same type of tumor as the primary site. Metastasis is the characteristic of colon cancer deterioration. Distant metastases from colon cancer are mainly in the liver, and about $50 \%$ of patients will have preoperative or postoperative liver metastases. About $30 \%$ of patients have insidious liver metastases that cannot be detected by ultrasound or CT before surgery. However, only a small percentage (10\%-20\%) are suitable for surgical resection, and $70 \%$ of them recur after surgery $(3,4)$. Therefore, it is crucial to find new treatments for colon cancer. 
Tumor cells live in a stressful environment with various important nutrients such as glucose, glutamine and oxygen in dynamic changes $(5,6)$. Therefore, their major biomolecules including polysaccharides, proteins, lipids, nucleic acid synthesis, and energy and NADPH production are altered to adapt to their survival and proliferation requirements (7). Otto-Warburg first observed in their experiments that tumor tissues can take up large amounts of glucose in the presence of adequate glucose and oxygen levels in the in vitro culture environment. Interestingly, however, pyruvate produced by tumor cells through glycolysis was not coupled to the tricarboxylic acid cycle (TCA cycle) in the mitochondria, but was converted to lactate and they called this phenomenon the 'Warburg effect' (8). Glutamine-fructose-6phosphate transaminase (GFPT) is the rate-limiting enzyme of the hexosamine biosynthetic pathway (HBP) and consists of the unlinked highly homologous genes GFPT1 and GFPT2 encoding the transaminase, one of the most common and important pathways in glucose metabolism responsible for glycosylation $(9,10)$. GFPTs catalyze the formation of glucosamine-6-phosphate from glucosamine and fructose-6-phosphate, and glucosamine infusion can bypass this pathway (11). Recent studies have shown that aberrant expression of GFPTs can lead to reprogramming of fibroblast metabolism in lung adenocarcinoma (12-14) and also GFPT2 is highly expressed in more aggressive breast cancer cell lines (11).

In this study, we found that GFPT2 was highly expressed in colon adenocarcinoma and that high expression was associated with poor pathological features and poor clinical prognosis. By analyzing the signaling pathways of the gene, we found that the gene mediates pathways related to tumor development, and more interestingly, the gene was associated with the tumor microenvironment and immune-related signaling pathways. Overall, our findings uncovered new evidence of functional properties associated with metabolic reprogramming in colon cancer, as well as potential new therapeutic pathways.

\section{Methods}

\section{Patients and tissue samples}

We obtained tissue samples from 83 colon cancer patients collected from the Affiliated Huaian No.1 People's Hospital of Nanjing Medical University. These tissue samples were collected from 2015 to 2021, and they all had complete clinicopathological data, and all samples were de-identified. These data included sex, age, cellular differentiation, TNM stage, primary tumor size, and lymph node metastasis status. All procedures in this study involving human material were approved by the committee of the Affiliated Huaian No.1 People's Hospital of Nanjing Medical University, and consent forms were acquired from all patients.

\section{Immunohistochemistry (IHC)}

The colon cancer tissues were embedded in paraffin and cut off in sections $(4 \mu \mathrm{m})$. The tissue samples were de-paraffined with xylene and hydrated with graded ethanol. Slices were treated with $1 \mathrm{X}$ citrate repair solution at $100^{\circ} \mathrm{C}$ for 30 minutes. Next, tissue sections were placed in $3 \%$ hydrogen peroxide for 15 
minutes at room temperature. Then, the sections were blocked with with $5 \%$ bovine serum albumin (BSA) (Sigma, A3294) for 1 hour at room temperature. The tissue sections were incubated overnight at $4^{\circ} \mathrm{C}$ with primary antibody (anti-GFPT2, Thermo Fisher Scientific, PA5-26290, 1:100 dilution). The next day, the sections were incubated with secondary antibody (Goat anti-Rabbit, Dako, P0448) at $37^{\circ} \mathrm{C}$ for 1 hour. Then DAB (DAB and Substrate Chromogen System, Dako) and hematoxylin staining of the nuclei were performed. The staining intensity was graded as follows: negative- $(<10 \%$ positive cells), positive+ (11\%-30\% positive cells); positive++ (31\%-50\% positive cells); positive+++ (51\%-100\% positive cells). Negative- and positive+ were considered as low GFPT2 expression, and positive++ and positive+++ were considered as high GFPT2 expression.

\section{Survival analysis}

We extracted colon adenocarcinoma (COAD) RNAseq data from The Cancer Gene Atlas (TCGA) dataset, log2 transformed the RNAseq data in Fregments Per Kilobase per Million (FPKM) formate. Then we removed normal tissues and merged GFPT2 expression and clinical information. We included age, excluded the effect of age on survival time, and performed statistical analysis of survival data through the survivor $R$ package and used it for visualization through the survminer $R$ package in 329 COAD samples.

\section{Gene oncology (GO) and KEGG analysis}

We extracted COAD RNAseq data from TCGA dataset, log2 transformed the RNAseq data in FPKM formate, and removed normal tissues. Next, we divided the samples into GFPT2 low and high expression groups and performed differential analysis to obtain differentially expressed genes $(P<0.05)$. GO and KEGG enrichment analysis was performed using the ClusterProfilter $\mathrm{R}$ package, and significant enrichment pathways were obtained ( $p$-values $<0.05$ ).

\section{Analysis of Stromal and Immune Cell Infiltration}

Stromal, immune cell, ESTIMATE and tumor purity scores were obtained by the ESTIMATE (Estimation of STromal and Immune in MAlignant Tumors Using Expression Data) algorithm (15). The stromal, immune scores were used to check the infiltrative expression of stromal and immune cells in COAD. Correlation of tumor-infiltrating stromal cell and immune cell characteristics and GFPT2 expression using Timer (Tumor Immune Estimation Resource) (http:// cistrome.org/TIMER/).

\section{Single Cell Analysis}

The Tumor Immune Single Cell Center (TISCH) (http://tisch.comp-genomics.org/) was used to study the expression of the GFPT2 gene in the tumor microenvironment as a single cell subset. TISCH is a scRNAseq database focused on the tumor microenvironment (TME). TISCH provides detailed at the single cell level annotation of cell types, allowing one to explore TME in different cancer types (16). In this dataset, there are three main cell types, including immune cells, stromal cells, and malignant cells. 


\section{GFPT2 co-expression heatmap analysis}

We extracted COAD RNAseq data from TCGA dataset, log2 transformed the RNAseq data in FPKM formate, and removed normal tissues. The GFPT2 expression, the expression levels of JAK STAT signaling pathway-related genes (JAK1, JAK2, JAK2, TYK2, STAT1, STAT2, STAT3, STAT4, STAT5A, STAT5B, STAT6) were obtained from COAD samples in TCGA dataset. The heatmap was visualized with ggplot2. Pearson correlation coefficient tests were used to estimate the association between the GFPT2 expression and other genes.

\section{Correlation with GFPT2 expression and immune cells}

Firstly, the contents of immune cells in each sample were calculated using CIBERSORT and the samples were filtered by $p<0.05$. Next, the correlation coefficient between GFPT2 expression and immune cells was calculated using the cor.test function in R language, and the correlation between gene expression and immune cells was verified using the spearman correlation test. If the $p$-value was $<0.001$, it indicated that the correlation between GFPT2 expression and immune cells was significant. The correlation graph was then visualized using the ggpubr $\mathrm{R}$ package.

\section{Corrplot of GFPT2 and other factors}

We extracted COAD RNAseq data from TCGA dataset, log2 transformed the RNAseq data in FPKM formate, and removed normal tissue. The GFPT2 expression and other factors (CAFs-associated factors, EMT-related factors, $T$ cell exhaustion factors, immunosuppressive factors) were obtained from COAD samples in TCGA dataset. The corrplot package was performed and the pearson correlation coefficient tests were used to estimate the association between the GFPT2 expression and other factors.

\section{Drug sensitivity analysis}

Gene expression and drug sensitivity data were downloaded from CellMiner dataset, and we removed drugs without clinical trials or FDA approval. The correlation coefficients between GFPT2 expression and drug sensitivity were calculated using the cor.test function in $\mathrm{R}$ language, and correlation tests were done. We defined $p<0.05$ as the correlation between the target gene and drug sensitivity was significant. If the correlation coefficient was greater than 0 , it means that there was a positive correlation between GFPT2 expression and drug sensitivity.

\section{Results}

\section{GFPT2 is highly expressed in colon cancer and correlates with poor patient pathological features.}

To explore the roles of GFPT2 in patients with colon cancer, we first investigated the expression levels of GFPT2 in colon cancer. We collected tissues from 83 colon cancer patients and detected the protein levels of GFPT2 by immunohistochemistry (IHC). Figure 1a shows typical images of GFPT2 expression levels in colon cancer tissues and corresponding paraneoplastic tissues. Our results showed that GFPT2 
was an oncogene highly expressed in colon cancer tissues compared to paraneoplastic tissues (Fig. 1a, 1b).

We further investigated the correlation between the expression levels of GFPT2 and the pathological characteristics of colon cancer patients. As shown in Table 1, high levels of protein expression of GFPT2 were positively correlated with unfavorable clinicopathological features in 83 samples of colon cancer patients. Specifically, increased GFPT2 levels were positively associated with advanced TNM $\left(\chi^{2}=7.803\right.$, $P=0.005<0.01)$, advanced primary tumor size $\left(X^{2}=5.896, P=0.015<0.05\right)$ and excessive lymph node metastasis $\left(X^{2}=10.008, P=0.007<0.01\right)$. However, protein expression of GFPT2 showed no correlation in patient's gender, age, and cell differentiation $(P>0.05)$. We show representative images of the protein levels of different GFPT2 (negative-, positive+, positive++, positive ++++) in Figure 1c. Our results show that more advanced TNM stages show higher GFPT2 expression levels than early TNM stages (Fig. 1d). Moreover, the different GFPT2 positive levels $(-,+,++,++++)$ in early TNM stages were $9.80 \%, 49.02 \%$, $33.33 \%$ and $7.85 \%$, respectively; and $3.12 \%, 34.38 \%, 37.50 \%$ and $25.00 \%$ in late TNM, respectively (Fig. $1 \mathrm{e})$. In addition, more cases of lymph node metastasis were positively correlated with higher GFPT2 levels (Fig. 1f). The positive levels of different GFPT2 $(-,+,++,++++)$ were $9.80 \%, 49.02 \%, 33.33 \%$ and $7.85 \%$ in N0 stage, respectively; and $5.26 \%, 52.63 \%, 21.05 \%$ and $21.06 \%$ in N1 stage, respectively; and $0.00 \%, 7.69 \%, 61.54 \%$ and $30.77 \%$ in N2 stage, respectively (Fig. 1g). 
Table 1

Association between GFPT2 protein expression and clinicopathological characteristics in colon cancer tissues.

\begin{tabular}{|c|c|c|c|c|c|}
\hline \multirow[t]{2}{*}{ Clinicopathological characteristic } & \multirow[t]{2}{*}{$\mathbf{n}$} & \multicolumn{2}{|c|}{ GFPT2 expression } & \multirow[t]{2}{*}{$\chi^{2}$} & \multirow[t]{2}{*}{$P$ value } \\
\hline & & Low expression & High expression & & \\
\hline Total number & 83 & 42 & 41 & & \\
\hline Gender & & & & 0.003 & 0.958 \\
\hline Male & 49 & $23(46.94)$ & $26(53.06)$ & & \\
\hline Female & 34 & $19(55.88)$ & $15(44.12)$ & & \\
\hline Age (years) & & & & 0.071 & 0.790 \\
\hline$<65$ & 38 & $21(55.26)$ & $17(44.74)$ & & \\
\hline$\geq 65$ & 45 & $21(46.67)$ & $24(53.33)$ & & \\
\hline Cell differentiation & & & & 2.049 & 0.359 \\
\hline Well & 21 & $9(42.86)$ & $12(57.14)$ & & \\
\hline Moderate & 36 & $19(52.78)$ & $17(47.22)$ & & \\
\hline Poor & 26 & $9(34.62)$ & $17(65.38)$ & & \\
\hline Primary tumor size & & & & 5.896 & $0.015^{\star}$ \\
\hline T1-2 & 48 & $30(62.50)$ & $18(37.50)$ & & \\
\hline T3-4 & 35 & $12(34.29)$ & $23(65.71)$ & & \\
\hline Lymph node metastasis & & & & 10.008 & $0.007^{\star \star}$ \\
\hline No & 51 & $32(62.75)$ & $19(37.25)$ & & \\
\hline N1 & 19 & $8(42.11)$ & $11(57.89)$ & & \\
\hline N2 & 13 & $2(15.38)$ & $11(84.62)$ & & \\
\hline TNM & & & & 7.803 & $0.005^{\star \star}$ \\
\hline $1 / I I$ & 51 & $32(62.75)$ & $19(37.25)$ & & \\
\hline III/IV & 32 & $10(31.25)$ & $22(68.75)$ & & \\
\hline
\end{tabular}

To further explore the relationship between GFPT2 expression and prognosis of colon cancer patients, we investigated the overall survival (OS) of GFPT2 in the TCGA database using Kaplan-Meier. The results showed that high GFPT2 expression was significantly associated with poorer OS in 329 patients with colon adenocarcinoma (COAD) $(P=0.028<0.05)(F i g .1 \mathrm{~h})$. We conclude that GFPT2 expression levels are 
associated with poor pathological characteristics and poor prognostic features in patients with colon cancer.

\section{GFPT2 is associated with tumor-associated enrichment pathways.}

To explore the expression and pathway enrichment of GFPT2 in the TCGA database, we analyzed GFPT2 and related genes in COAD cases. We performed Gene oncology (GO) analysis using the ClusterProfiler R package and obtained GFPT2 significantly enriched functions and pathways $(p<0.05)$. We displayed 30 pathways significantly enriched with GFPT2 that were associated with many tumor-related pathways, including pathways in cancer, Focal adhesion, Adhersion molecules cams, ECM receptor interaction, JAKSTAT signaling pathway and MAPK signaling pathway (Fig. 2a-e), suggesting that GFPT2 may play an important function in tumorigenesis and progression. In cell biology, focal adhesion and ECM receptor interaction mediate the regulation of cell adhesion to the extracellular matrix (ECM) mainly through its transmission between the ECM and interacting cells $(17,18)$. They, therefore, play a central role in cell migration (18). Increased GFPT2 levels were positively linked to the enrichment of focal adhesion and ECM receptor interaction (Fig. 2b, 2c), indicating that GFPT2 may have the essential position in stromal cell and invasive of tumors.

The JAK-STAT signaling pathway is an important pathway in cell biology that is involved in various vital physiological functions, including immunity, cell death and tumor formation (19). Abnormalities in JAKSTAT signaling will lead to various diseases, such as skin diseases, cancer and diseases affecting the immune system (20). It has four JAK proteins, namely JAK1, JAK2, JAK3 and TYK2 (20). Seven STAT proteins are involved, which can be identified as STAT1, STAT2, STAT3, STAT4, STAT5A, STAT5B and STAT6 (20). We demonstrated a remarkable positively related correlation between GFPT2 overexpression and proteins associated with the JAK-STAT signaling pathway (Fig. 2f). JAK-STAT signaling is able to interconnect with other cellular signaling pathways, such as the MAPK/ERK pathway. JAKs phosphorylated receptors can bind to the $\mathrm{SH} 2$ binding domain of Grb2, an important protein in the MAPK/ERK pathway (19). In addition, MAPK (mitogen-activated protein kinase), can phosphorylate STATs, thus allowing STATs to increase gene transcription, which in turn promotes the JAK-STAT signaling pathway (19). Interestingly, our results revealed that an increase in GFPT2 promotes the MAPK signaling pathway (Fig. 2e), a result consistent with previous published reports.

Interestingly, in addition to GFPT2 high expression enriching lots of tumor-related pathways, increased GFPT2 expression also enriched a large number of immune-related pathways, including Chemokine signaling pathway, Cytokine cytokine receptor interaction, Toll-like receptor signaling pathway, NOD-like receptor signaling pathway, Natural killer cell mediated cytotoxicity, B cell receptor signaling pathway and T cell receptor signaling pathway (Fig. 2a).

\section{Association of GFPT2 levels with tumor microenvironment}

We have known that GFPT2 expression related to ECM and immune pathways, since stromal cells and immune cells are major components of the tumor microenvironment (TEM), so we speculated whether 
GFBP2 would be involved in TEM. To confirm our hypothesis, we observed the correlation of GFPT2 expression with stromal cell and immune cell infiltrations. Our results revealed that increased GFPT2 expression was strongly and positively associated with stromal score $(R=0.89, P<0.0001)$ (Fig. 3a), immune score $(R=0.64, P<0.0001)$, and ESTIMATE score (a combined score of stromal and immune cells) $(R=0.82, P<0.0001)$ (Fig. 3a-c), indicating that GFPT2 expression affects stromal and immune cell infiltrations. Interestingly, the high levels of GFPT2 were significantly and negatively linked to tumor purity $(R=0.82, P<0.0001)$ (Fig. $3 d)$, suggesting that GFPT2 mainly affects the predominant activity of TEM, which has essential position in stromal cell and immune cell infiltrations.

\section{Correlation between GFPT2 levels and stromal cell infiltration.}

The TEM is composed of ECM, cancer-associated fibroblasts (CAFs), myofibroblasts, immune cells and other factors (21). In order to examine the relationship between GFPT2 levels and stromal cell infiltrations, we first surveyed the Tumor Immune Single-cell Hub (TISCH) (http://tisch.compgenomics.org/) database (a single cell center) to investigate which cell subpopulations of GFPT2 are primarily expressed in. We explored a colon cancer single cell GSE dataset (GSC_GSE146771_Smartseq2) and found that GFPT2 was expressed in both immune and stromal cell single cell subpopulations (Fig. 4a-c). Since the tumor-associated stromal cells mainly include endothelial cells, fibroblasts and myofibroblasts. We next investigated which stromal cell components were the cell subpopulations with high GFPT2 expression in colon cancer. The findings indicated that fibroblasts were the major GFPT2 expressing cells, and the expression of GFPT2 was very high in fibroblasts compared to other cell subpopulations (Fig. 4c), indicating that GFPT2 has an important function in CAFs.

Next, we inspected the association among GFPT2 mRNA expression and biomarkers associated with CAFs. CAFs primarily express a-smooth muscle actin (a-SMA), fibronectin (FAP), cytoskeletal protein (Palladin), mucin-type protein (podoplanin), and prolyl 4-hydroxylase, while TGF- $\beta$, PDGF, HGF, FGF2 and THBS1 are the main factors that promote the activation of CAFs (22-24). S100A8/A9 could facilitate the proliferation of fibroblasts and worked in the differentiation of fibroblasts to myofibroblasts (25). In the current study, we noticed that the expression of GFPT2 had significantly positive correlation with the above mentioned markers related to CAFs (Fig. 4d).

CAFs exert huge contributions in tumorigenesis and development, which can mainly boost angiogenesis, promote the initiation of epithelial-mesenchymal transition (EMT) and affect the survival of tumor cells (26). To confirm the effects of GFPT2 expression on EMT, we examined the levels of GFPT2 in relation to EMT-related markers. The results showed that GFPT2 expression was highly correlated with EMT-related factors (Fig. 4e).

\section{Association of GFPT2 levels with immune cell infiltration.}

To better evaluate the influences of GFPT2 levels on immune cell infiltration, we calculated the correlation coefficients between GFPT2 expression and immune cells using the CIBERSORT method. The correlation between GFPT2 expression and immune cells was verified by spearman's correlation test. There was a 
statistically significant positive correlation between increasing GFPT2 and immune cell fractions, including Macrophages M0, Neutrophils and activated Mast cells (Fig. 5a-C). However, overexpression of GFPT2 adversely related to certain immune cell components, such as Monocytes, resting Mast cells, T cells follicular helper, plasma cells, activated CD4 T cells memory, activated NK cells, resting Dendritic cells, CD8 T cells (Fig. 5a-c).

In general, T-cell depletion is vital factor for the effectiveness of immune checkpoint blockade (27). To further discuss the relevance of GFPT2 levels to marker genes of T-cell exhaustion, our results displayed a remarkable association of GFPT2 levels with LAG3, BTLA, PDCD1, TIGIT and CTLA4 (Fig. 5d). Our analysis suggested that GFPT2 was mainly positively correlated with immunosuppressive cells, such as fibroblasts and macrophages, and therefore, we hypothesized that GFPT2 might be involved in the regulation of COAD as an immunosuppressive component. To verify this point, we conducted a correlation analysis between GFPT2 expression and key factors of immunosuppression. The results showed that GFPT2 levels were significantly and positively correlated with many immunosuppressive factors (Fig. 5e).

\section{The relationship between GFPT2 expression levels and drug sensitivity.}

We downloaded gene expression and drug sensitivity data from CellMiner and removed drugs without clinical trials or FDA approval and calculated the correlation coefficient between GFPT2 expression and drug sensitivity using the cor.test function and correlation in $\mathrm{R}$ language. We selected the top 16 drugs associated with GFPT2 by $\mathrm{R}$ value, and the results showed that GFPT2 expression was associated with a number of drugs, including Deforolimius, SGX-523, JNJ-38877605, Motesanib, Staurosporine, Itraconazole, CCT-128930, AZD-5363, AS-703569, AT-9283, Silmitasertib, Rigosertib, LY-294002, Rebimastat, PF-04217903 (Fig. 6), and the higher GFPT2 expression was linked to better sensitivity of tumor cells to these drugs. And the expression level of GFPT2 was associated with increased resistance to By-Product of CUDC-305 (Fig. 6). These results suggest that the expression of GFPT2 may be a judgment of the sensitivity of a certain class of drugs.

\section{Discussion}

GFPT2, the rate-limiting enzyme of HBP, plays an important role in the metabolic activity of cells, especially in their glucose metabolism (28). In our study, we found that GFTP2 was aberrantly expressed in CRC tumor tissues relative to normal tissues and that GFTP2 expression and clinical TNM staging as well were positively correlated, and analysis of the TCGA database also revealed that patients with high GFPT2 expression had decreased survival time. These results suggest that GFPT2 plays a facilitating role in the progression of colon cancer.

By analyzing the KEGG and GO signaling pathways, we found that GFTP2 affects the development of colon cancer mainly through Focal adhesion, and ECM receptor interaction. Both of these are related to cell migration and interactions between receptors of extracellular mechanisms $(29,30)$. Focal adhesion kinases (FAKs) are a class of cytoplasmic non-receptor protein tyrosine kinases that belong to the protein 
tyrosine kinase (PTK) superfamily and are therefore also known as PTK II (30). FAKs can integrate signals from integrins, growth factors and mechanical stimuli to activate intracellular PI3K/Akt, Ras/MAPK and other signaling pathways to regulate cell growth (31-33). Numerous studies on the association of FAKs with different types of cancers have shown a close link between FAKs and the biological mechanisms that promote cancer development and progression (34). Moreover, FAKs tend to be inversely associated with better clinical cancer sample outcomes, and related studies have found that FAKs are overexpressed and/or hyperphosphorylated in a variety of cancer cells and are responsible for cell migration, survival, proliferation and adhesion $(35,36)$. Recently studys found that FAKs promote renewal and drug resistance in cancer stem cells (CSCs) by acting in survival signaling (37). For example, FAKs and extracellular signal-regulated kinase (ERK1/2) pathways are involved in regulating the growth and metastasis of liver cancer stem cells (38).

ECM is an essential component of stromal cells, therefore, we hypothesized that GFPT2 might have regulatory effects on TEM, since stromal cells were a critical component of TEM (39). Cancer development and progression often coincide with changes in the surrounding stroma (40). Cancer cells can functionally shape the microenvironment by secreting different chemokines and chemotactic factors (41). Once the microenvironment is formed, this leads to a reprogramming of the surrounding cells, which enables them to play a crucial function in the survival and progression of the tumors (42). Interestingly, in the current study, increased GFPT2 expression was highly associated with stromal cells, especially CAFs, we also found that the expression of GFPT2 had significantly positive correlation with CAFs-related factors.

During cancer progression, epithelial tumors may undergo EMT, which alters the characteristics of tumor cells, resulting in the loss of the epithelial marker E-calcineurin and an increase in the mesenchymal markers $\mathrm{N}$-calcineurin, fibronectin, and wave proteins $(43,44)$. Numerous reports have shown that CAFs and tumor-associated macrophages (TAMs) can contribute to cancer cell adhesion and growth, playing critical roles in ECM alterations (45). This process enhances the separation of cancer cells from the primary tumors and enhances invasiveness, thus allowing cancer cells to enter the bloodstream or lymph and eventually lead to distant metastasis (46). Here, GFPT2 expression was highly correlated with EMTrelated factors, indicating that GFPT2 might be capable of regulating the promotion of colon cancer metastasis. This speculation could be confirmed to certain extent by the high expression of GFPT2 with more lymph node metastasis. However, we will perform some cellular and animal experiments in the future to further confirm whether GFPT2 is associated with colon cancer.

Immune cells are another important component of TEM, and a growing number of evidences suggest that the presence of innate immune cells (macrophages, neutrophils, dendritic cells, and natural killer cells, etc.) as well as adaptive immune cells (T cells and B cells) in TME promoter tumor progression (40). In this study, increasing GFPT2 expression was significantly associated with immune cells, and positively correlated with immunosuppressive cells and T-cell exhaustion. Therefore, GFTP2 may be involved in tumor cell immune escape. Currently, immunotherapies targeting immune checkpoints, such as anti-PDL1 antibodies, have shown clinical activity against various types of cancer, and the increase in immune 
checkpoints can suppress the anti-tumor immune response of T cells (47). Inhibition of the antitumor immune response of T cells by increasing the expression of PD-1 and CTLA4 receptors makes GFTP2 a potential new target for immunotherapy.

However, how does GFPT2 modulate the role of immune cells? The JAK-STAT signaling pathway mediates numerous tumor immunomodulatory processes, including tumor cell recognition and tumor immune escape (48). The anti-tumor immune response is dominated by two of these factors, STAT1 and STAT2, which act by inducing type I and type II interferons (IFN) (49). Conversely, STAT3 mainly maintains cancer cells survival and regulates immunosuppression and continuous inflammation in TEM (50). In this study, we demonstrated a remarkable positively related correlation between GFPT2 overexpression and proteins associated with the JAK-STAT signaling pathway. Therefore, we speculate that GFPT2 may mediate the regulation of immunosuppression mainly through the JAK STAT signaling pathway. In the future, we will design some animal experiments to confirm our speculations.

\section{Conclusions}

In this study, we found that GFTP2 and colon cancer are closely related, and we identified the value of GFTP2 in patient prognosis prediction, tumor microenvironment, tumor immunity and drug sensitivity through the analysis of TCGA database and validation of clinical samples. Currently, the specific underlying mechanism between GFTP2 expression and tumor immunity in colon cancer remains unclear, which deserves further investigation. Overall, our work largely revealed the roles of GFTP2 in tumorigenesis, especially in immune response, tumor microenvironment and drug resistance, which is crucial for the development of customized cancer therapies.

\section{Abbreviations}

GFPT2: Glutamine-fructose-6-phosphate transaminase 2;

IHC: immunohistochemistry; OS: overall survival;

TCGA: The Cancer Gene Atlas;

TISCH: The Tumor Immune Single Cell Center;

COAD: colon adenocarcinoma;

GO: Gene oncology;

TEM: tumor microenvironment;

TCA: tricarboxylic acid;

HBP: hexosamine biosynthetic pathway; 
FPKM: Fregments Per Kilobase per Million;

ESTIMATE: Estimation of STromal and Immune in MAlignant Tumors Using Expression;

ECM: extracellular matrix;

CAFs: cancer-associated fibroblasts;

a-SMA: a-smooth muscle actin;

FAP: fibronectin;

EMT: epithelial-mesenchymal transition;

FAKs: Focal adhesion kinases;

PTK: protein tyrosine kinase;

CSCs: cancer stem cells; IFN: interferons.

\section{Declarations}

\section{Ethical approval and consent of participate}

All procedures in this study involving human material were approved by the committee of the Affiliated Huaian No.1 People's Hospital of Nanjing Medical University, and consent forms were acquired from all patients.

\section{Consent for publication}

Not Applicable.

\section{Availability of data and materials}

The datasets used and/or analyzed during the current study are available from the corresponding authors upon reasonable request.

\section{Competing interests}

The authors have no conflict of interest to declare.

\section{Funding}

Not Applicable.

\section{Authors' contribution}


$X D$ and $X Z$ designed the study. XD and HL performed the IHC experiment. YY and QZ analyzed the data. $X D, H L$ and $X Z$ discussed the project. $X D$ and $H L$ drafted, and $X Z$ proofread and revised the manuscript. All authors read and approved the final manuscript.

\section{Acknowledgments}

We would like to thank the TISCH database for open access to the Colon cancer sequencing datasets.

\section{References}

1. Liu Y, Wu K, Lai H, Zeng Z, Zhang BJJoX-rs, technology. Clinical application of fluoroscopic guided percutaneous antegrade ureteral stents placement for the treatment of malignant ureteral obstruction. 2019;27(3):453-60.

2. Barkhatov L, Aghayan DL, Scuderi V, Cipriani F, Fretland ÅA, Kazaryan AM, et al. Long-term oncological outcomes after laparoscopic parenchyma-sparing redo liver resections for patients with metastatic colorectal cancer: a European multi-center study. 2021:1-8.

3. Khan A, Fahl Mar K, Gokul S, Brown WAJTWJoBP. Mortality during US FDA clinical trials in patients with diabetes, hypertension, depression and schizophrenia. 2020;21(1):64-71.

4. Lin T, Yu J, Hu Y, Liu H, Lu Y, Zhao M, et al. Preliminary experience of dual-port laparoscopic distal gastrectomy for gastric cancer. 2019;22(1):35-42.

5. Cluntun AA, Lukey MJ, Cerione RA, Locasale JWJTic. Glutamine metabolism in cancer: understanding the heterogeneity. 2017;3(3):169-80.

6. Stincone A, Prigione A, Cramer T, Wamelink MM, Campbell K, Cheung E, et al. The return of metabolism: biochemistry and physiology of the pentose phosphate pathway. 2015;90(3):927-63.

7. Gu L, Casey JLL, Andrabi SA, Lee JH, Meza-Perez S, Randall TD, et al. Mitochondrial calcium uniporter regulates PGC-1a expression to mediate metabolic reprogramming in pulmonary fibrosis. 2019;26:101307.

8. Martínez-Reyes I, Chandel NSJNc. Mitochondrial TCA cycle metabolites control physiology and disease. 2020;11(1):1-11.

9. Kato N, Dasgupta R, Smartt C, Christensen BJImb. Glucosamine: fructose-6-phosphate aminotransferase: gene characterization, chitin biosynthesis and peritrophic matrix formation in Aedes aegypti. 2002;11(3):207-16.

10. Bueno M, Calyeca J, Rojas M, Mora ALJRb. Mitochondria dysfunction and metabolic reprogramming as drivers of idiopathic pulmonary fibrosis. 2020;33:101509.

11. Simpson NE, Tryndyak VP, Beland FA, Pogribny IPJBcr, treatment. An in vitro investigation of metabolically sensitive biomarkers in breast cancer progression. 2012;133(3):959-68.

12. Tian L, Wu D, Dasgupta A, Chen K-H, Mewburn J, Potus F, et al. Epigenetic metabolic reprogramming of right ventricular fibroblasts in pulmonary arterial hypertension: a pyruvate dehydrogenase kinase- 
dependent shift in mitochondrial metabolism promotes right ventricular fibrosis. 2020;126(12):1723-45.

13. Zhao H, Dennery PA, Yao HJAJoP-LC, Physiology M. Metabolic reprogramming in the pathogenesis of chronic lung diseases, including BPD, COPD, and pulmonary fibrosis. 2018;314(4):L544-L54.

14. Zhang H, Jia Y, Cooper JJ, Hale T, Zhang Z, Elbein SCJTJoCE, et al. Common variants in glutamine: fructose-6-phosphate amidotransferase 2 (GFPT2) gene are associated with type 2 diabetes, diabetic nephropathy, and increased GFPT2 mRNA levels. 2004;89(2):748-55.

15. Yoshihara K, Shahmoradgoli M, Martínez E, Vegesna R, Kim H, Torres-Garcia W, et al. Inferring tumour purity and stromal and immune cell admixture from expression data. 2013;4(1):1-11.

16. Sun D, Wang J, Han Y, Dong X, Ge J, Zheng R, et al. TISCH: a comprehensive web resource enabling interactive single-cell transcriptome visualization of tumor microenvironment. 2021;49(D1):D1420D30.

17. Chen CS, Alonso JL, Ostuni E, Whitesides GM, Ingber DEJB, communications br. Cell shape provides global control of focal adhesion assembly. 2003;307(2):355-61.

18. Kerrisk ME, Cingolani LA, Koleske AJJPibr. ECM receptors in neuronal structure, synaptic plasticity, and behavior. 2014;214:101-31.

19. Rawlings JS, Rosler KM, Harrison DAJJocs. The JAK/STAT signaling pathway. 2004;117(8):1281-3.

20. Aaronson DS, Horvath CMJS. A road map for those who don't know JAK-STAT. 2002;296(5573):1653-5.

21. Jung H-Y, Fattet $L$, Yang JJCCR. Molecular pathways: linking tumor microenvironment to epithelialmesenchymal transition in metastasis. 2015;21(5):962-8.

22. Kalluri RJNRC. The biology and function of fibroblasts in cancer. 2016;16(9):582-98.

23. Kalluri R, Zeisberg MJNRC. Fibroblasts in cancer. 2006;6(5):392-401.

24. Rönty MJ, Leivonen S-K, Hinz B, Rachlin A, Otey CA, Kähäri V-M, et al. Isoform-specific regulation of the actin-organizing protein palladin during TGF- $\beta 1$-induced myofibroblast differentiation. 2006;126(11):2387-96.

25. Araki K, Kinoshita R, Tomonobu N, Gohara Y, Tomida S, Takahashi Y, et al. The heterodimer S100A8/A9 is a potent therapeutic target for idiopathic pulmonary fibrosis. 2021;99(1):131-45.

26. Huang L, Xu A-M, Liu S, Liu W, Li T-JJWjogW. Cancer-associated fibroblasts in digestive tumors. 2014;20(47):17804.

27. Blank CU, Haining WN, Held W, Hogan PG, Kallies A, Lugli E, et al. Defining 'T cell exhaustion'. 2019;19(11):665-74.

28. Liu L, Pan Y, Ren X, Zeng Z, Sun J, Zhou K, et al. GFPT2 promotes metastasis and forms a positive feedback loop with p65 in colorectal cancer. 2020;10(8):2510.

29. Zhao X, Guan J-LJAddr. Focal adhesion kinase and its signaling pathways in cell migration and angiogenesis. 2011;63(8):610-5. 
30. Zhou J, Yi Q, Tang LJJoE, Research CC. The roles of nuclear focal adhesion kinase (FAK) on Cancer: a focused review. 2019;38(1):1-11.

31. Katz M, Amit I, Yarden YJBeBA-MCR. Regulation of MAPKs by growth factors and receptor tyrosine kinases. 2007;1773(8):1161-76.

32. Fan $X$, Xie $X$, Yang $M$, Wang $Y, W u ~ H$, Deng T, et al. YBX3 Mediates the Metastasis of Nasopharyngeal Carcinoma via PI3K/AKT Signaling. 2021;11:454.

33. Guo J, Li R, Xu Z, Tian P, Wang R, Li Y, et al. Upregulated Inc-HZO2 and miR-hz02 inhibited migration and invasion by downregulating the FAK/SRC/PI3K/AKT pathway in BPDE-treated trophoblast cells. 2021:e22757.

34. Adachi M, Hoshino Y, Izumi Y, Sakai H, Takagi SJCJoVR. Effects of inhibitors of vascular endothelial growth factor receptor 2 and downstream pathways of receptor tyrosine kinases involving phosphatidylinositol 3-kinase/Akt/mammalian target of rapamycin or mitogen-activated protein kinase in canine hemangiosarcoma cell lines. 2016;80(3):209-16.

35. Montor WR, Salas AROSE, de Melo FHMJMc. Receptor tyrosine kinases and downstream pathways as druggable targets for cancer treatment: the current arsenal of inhibitors. 2018;17(1):1-18.

36. Grant S, Qiao L, Dent PJFB. Roles of ERBB family receptor tyrosine kinases, and downstream signaling pathways, in the control of cell growth and survival. 2002;7(3762):389.

37. Brunton VG, Avizienyte E, Fincham VJ, Serrels B, Metcalf CA, Sawyer TK, et al. Identification of Srcspecific phosphorylation site on focal adhesion kinase: dissection of the role of $\mathrm{Src} \mathrm{SH} 2$ and catalytic functions and their consequences for tumor cell behavior. 2005;65(4):1335-42.

38. Jee BK, Surendran S, Park KM, Lee WK, Han CW, Kim YY, et al. Role of tumor necrosis factor-a, interleukin-8, and dexamethasone in the focal adhesion kinase expression by human nucleus pulposus cells. 2007;32(1):30-5.

39. Denton AE, Roberts EW, Fearon DTJSI. Stromal cells in the tumor microenvironment. 2018:99-114.

40. Thakkar S, Sharma D, Kalia K, Tekade RKJAb. Tumor microenvironment targeted nanotherapeutics for cancer therapy and diagnosis: A review. 2020;101:43-68.

41. Maacha S, Bhat AA, Jimenez L, Raza A, Haris M, Uddin S, et al. Extracellular vesicles-mediated intercellular communication: roles in the tumor microenvironment and anti-cancer drug resistance. 2019;18(1):1-16.

42. Hinshaw DC, Shevde LAJCr. The tumor microenvironment innately modulates cancer progression. 2019;79(18):4557-66.

43. Pradella D, Naro C, Sette C, Ghigna CJMc. EMT and stemness: flexible processes tuned by alternative splicing in development and cancer progression. 2017;16(1):1-19.

44. Kang K-W, Lee M-J, Song J, Jeong J-Y, Kim Y-K, Lee C, et al. Overexpression of goosecoid homeobox is associated with chemoresistance and poor prognosis in ovarian carcinoma. 2014;32(1):189-98.

45. Paolillo M, Schinelli SJljoms. Extracellular matrix alterations in metastatic processes. 2019;20(19):4947. 
46. Jolly MK, Ware KE, Gilja S, Somarelli JA, Levine HJMo. EMT and MET: necessary or permissive for metastasis? 2017;11(7):755-69.

47. Topalian SL, Taube JM, Anders RA, Pardoll DMJNRC. Mechanism-driven biomarkers to guide immune checkpoint blockade in cancer therapy. 2016;16(5):275-87.

48. Owen KL, Brockwell NK, Parker BSJC. JAK-STAT signaling: a double-edged sword of immune regulation and cancer progression. 2019;11(12):2002.

49. Groner B, von Manstein VJM, endocrinology c. Jak Stat signaling and cancer: Opportunities, benefits and side effects of targeted inhibition. 2017;451:1-14.

50. Jones LM, Broz ML, Ranger JJ, Ozcelik J, Ahn R, Zuo D, et al. STAT3 establishes an immunosuppressive microenvironment during the early stages of breast carcinogenesis to promote tumor growth and metastasis. 2016;76(6):1416-28.

\section{Figures}


a
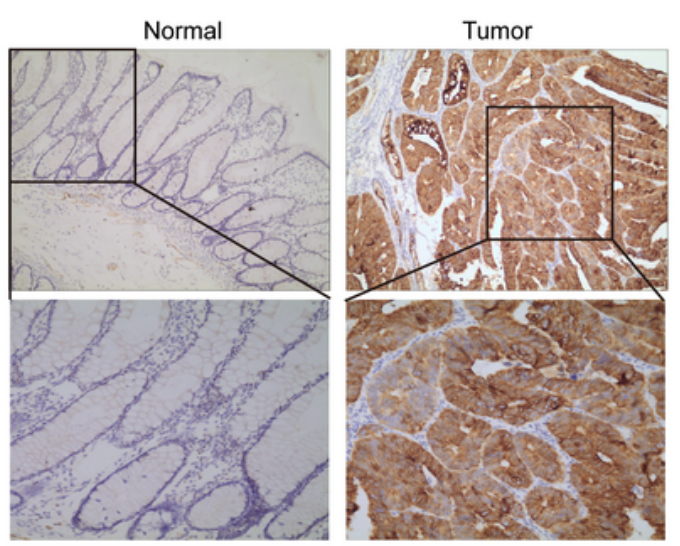

b

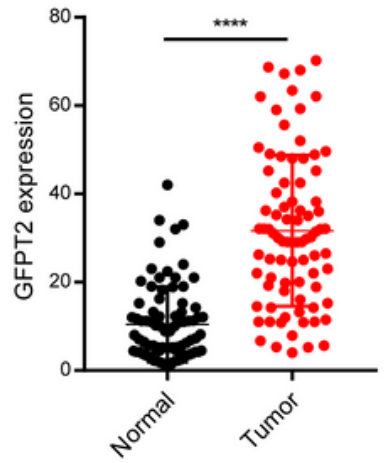

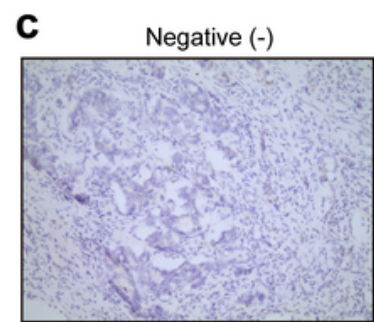

d

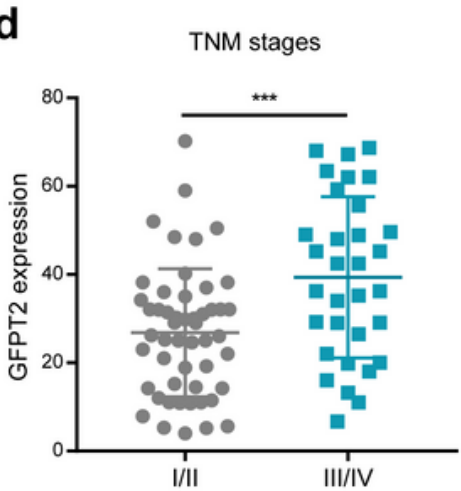

g

Nodal metastasis status

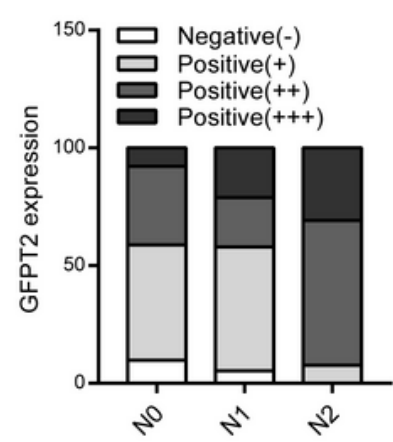

Positive (+)

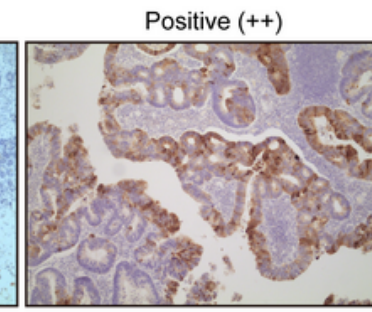

e

TNM stages
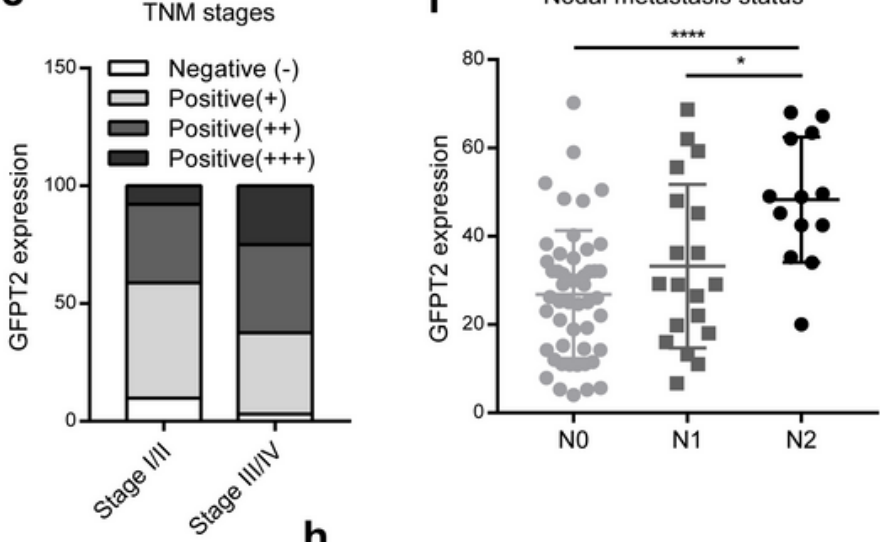

h

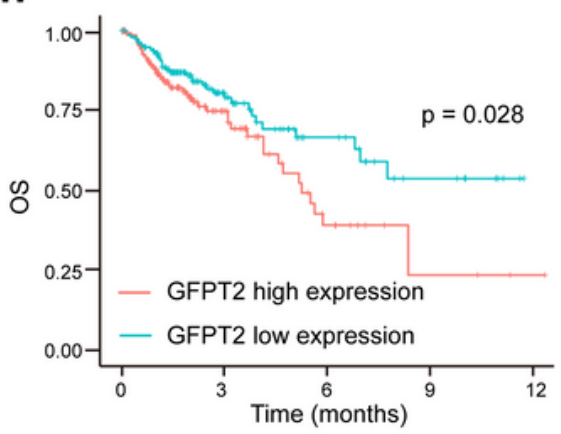

Figure 1

\section{Figure 1}

GFPT2 is highly expressed in colon cancer and correlates with poor patient pathological features. a The typical images of GFPT2 expression levels in colon cancer tissues and corresponding paracancerous tissues by immunohistochemistry (IHC). b GFPT2 was shown to be significantly higher in colon cancer tissues compared to paracancerous tissues. c Representative images of different GFPT2 (negative-, positive+, positive++, positive ++++) protein levels by IHC. d GFPT2 protein levels from different TNM 
stages (I/II stage or III/IV stage) in 83 colon cancer tissues. e The different GFPT2 positive levels $(-,+,++$, $++++)$ in $\mathrm{I} / \mathrm{Il}$ stages were $9.80 \%, 49.02 \%, 33.33 \%$ and $7.85 \%$, respectively; and $3.12 \%, 34.38 \%, 37.50 \%$ and $25.00 \%$ in III/IV stage, respectively. f GFPT2 protein levels from different NODE metastasis status (N0, N1, N2) in 83 colon cancer tissues. $g$ The positive levels of different GFPT2 $(-,+,++,++++)$ were $9.80 \%$, $49.02 \%, 33.33 \%$ and $7.85 \%$ in N0 stage, respectively; and $5.26 \%, 52.63 \%, 21.05 \%$ and $21.06 \%$ in N1 stage, respectively; and $0.00 \%, 7.69 \%, 61.54 \%$ and $30.77 \%$ in N2 stage, respectively. $\mathrm{h}$ The overall survival (OS) of GFPT2 in the TCGA database was used by Kaplan-Meier in 329 COAD samples from TCGA dataset. ${ }^{*} \mathrm{P}<0.05 ; * * * \mathrm{P}<0.001 ; * * * * \mathrm{P}<0.0001$.

a

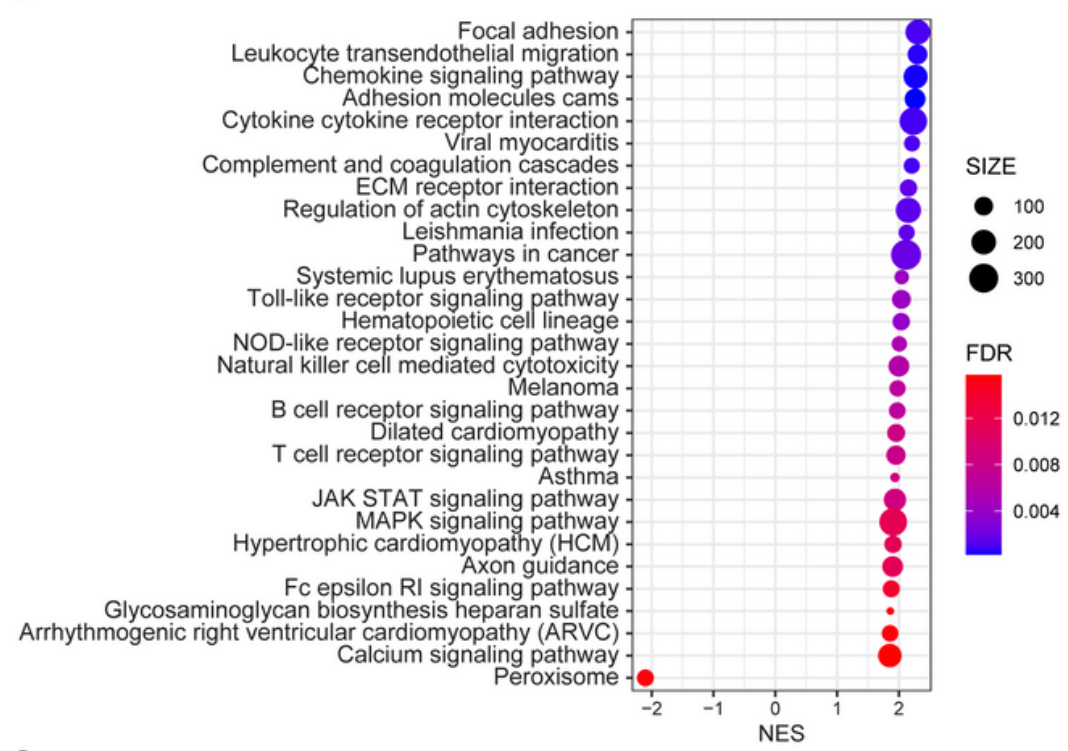

b

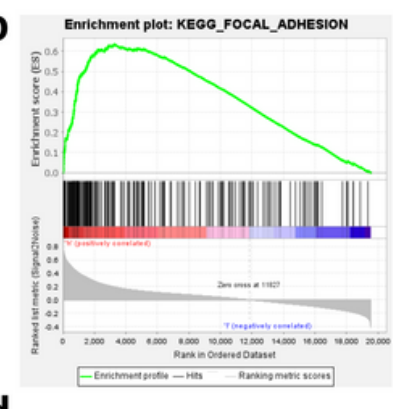

d

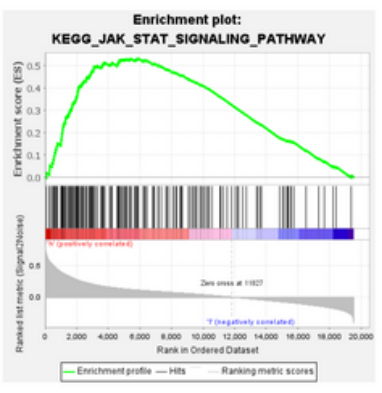

C

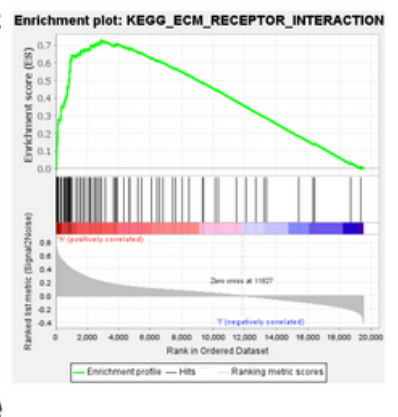

e

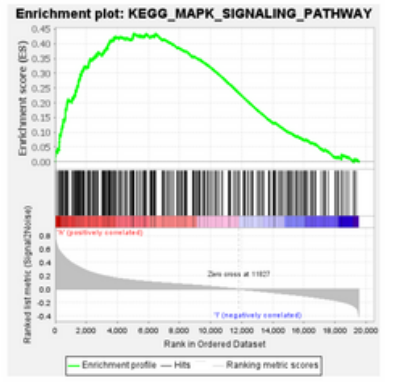

f

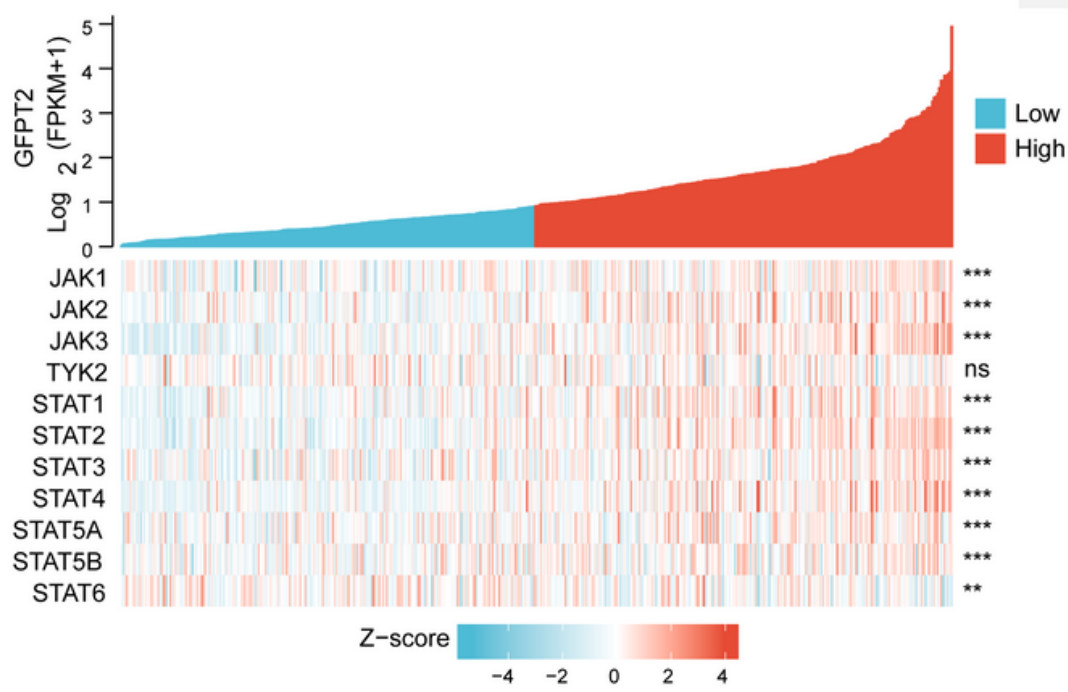

Figure 2

\section{Figure 2}

GFPT2 is associated with tumor-associated enrichment pathways. a-e KEGG enrichment pathways were performed using the clusterProfilter $R$ package. $f$ Heat map showing the relationship between GFPT2 and JAK-STAT signaling pathway-related genes. ${ }^{* * P}<0.01 ; * * * P<0.001 ;$ ns, no significance. 

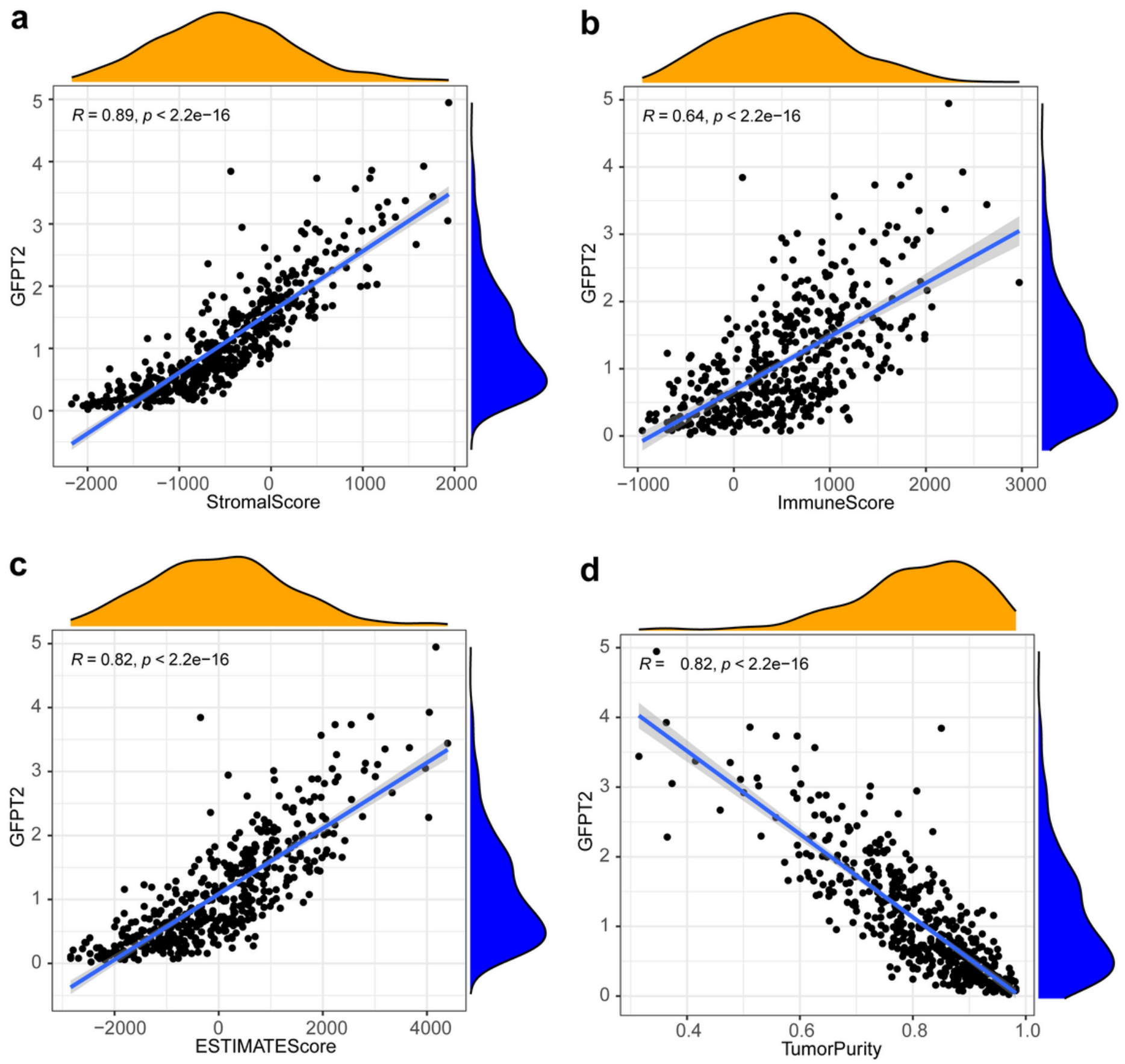

Figure 3

Figure 3

Association of GFPT2 levels with tumor microenvironment. The association between GFPT2 expression levels and stromal score (a), immune score (b), and ESTIMATE score (c) and tumor purity score (d). 
a

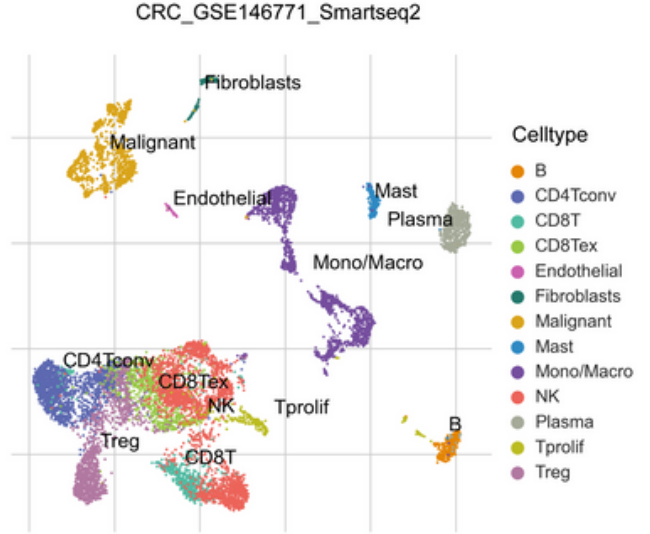

b

GFPT2

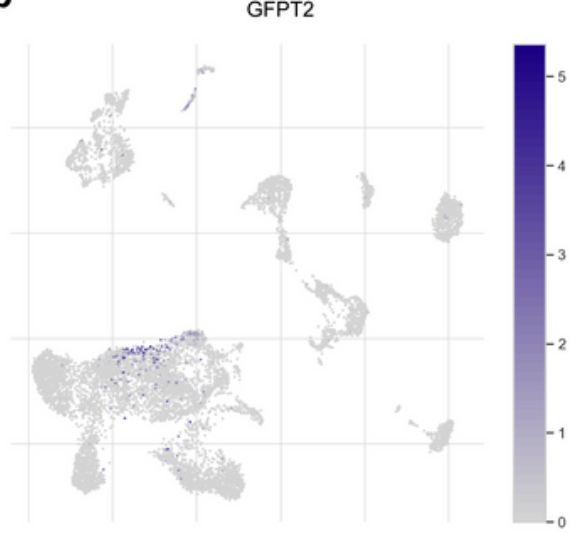

C

CRC_GSE146771_Smartseq2

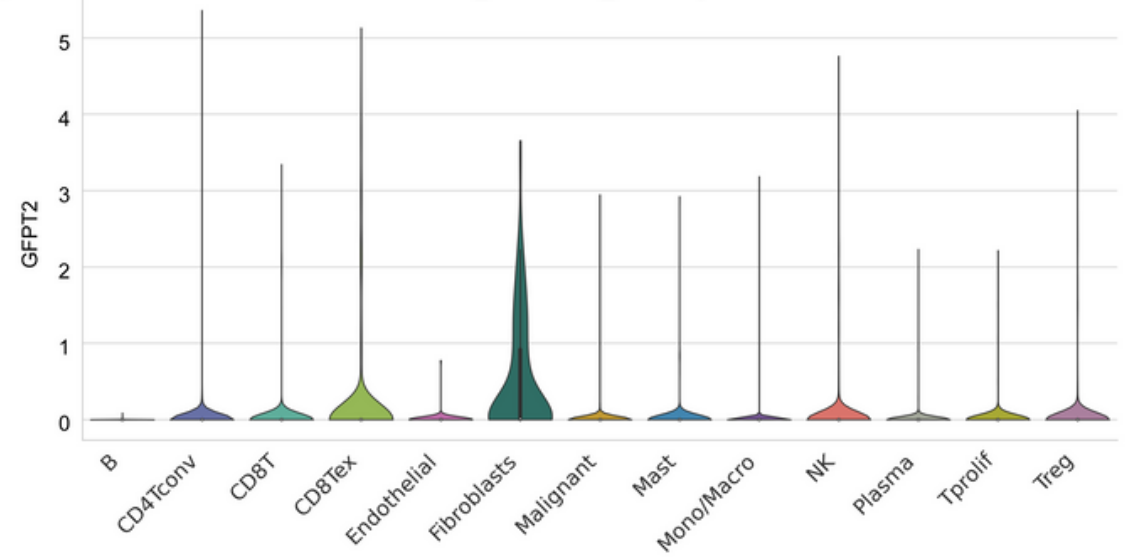

d

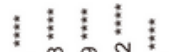

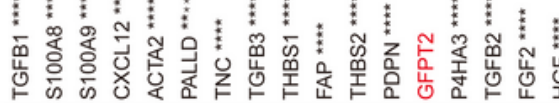

TGFB1

S100A8

S100A9

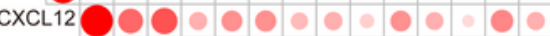
ACTA2 OOOOOOOOOOOO PALLDOOOOOOOOOOOOO TNC OOOOOOOO O TGFB3 OOOOOOOOOO THBS1 OOOOOOOO FAP OOOOO O THBS2 OOOOOO PDPN OOO GFPT2 $O O O$ P4HA3 $\bigcirc \bigcirc O$ TGFB2 $\bigcirc \bigcirc$ FGF2 $\bigcirc$ HGF e Corrplot of GFPT2 and EMT-related factors

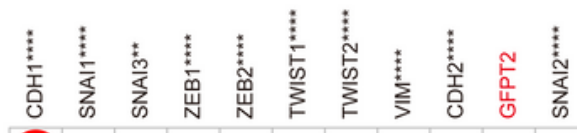

$\mathrm{CDH} 1$

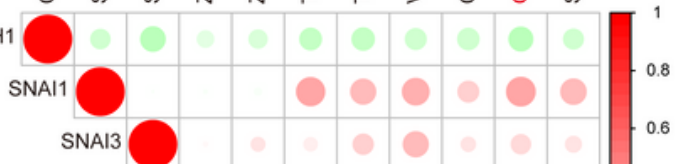

ZEB
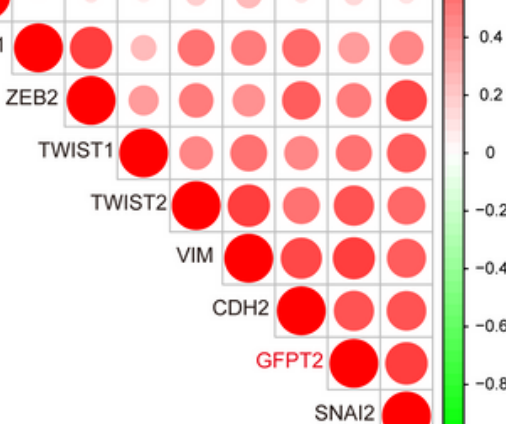

Figure 4

\section{Figure 4}

Correlation between GFPT2 levels and stromal cell infiltration. a-c GFPT2 was expressed in both immune and stromal cell single cell subpopulations in a colon cancer single cell GSE dataset (GSC_GSE146771_Smartseq2) from TISCH dataset. d Corrplot was used to perform the correlation between GFPT2 levels and CAFs-associated factors. e Corrplot was used to perform the correlation between GFPT2 levels and EMT-related factors. 

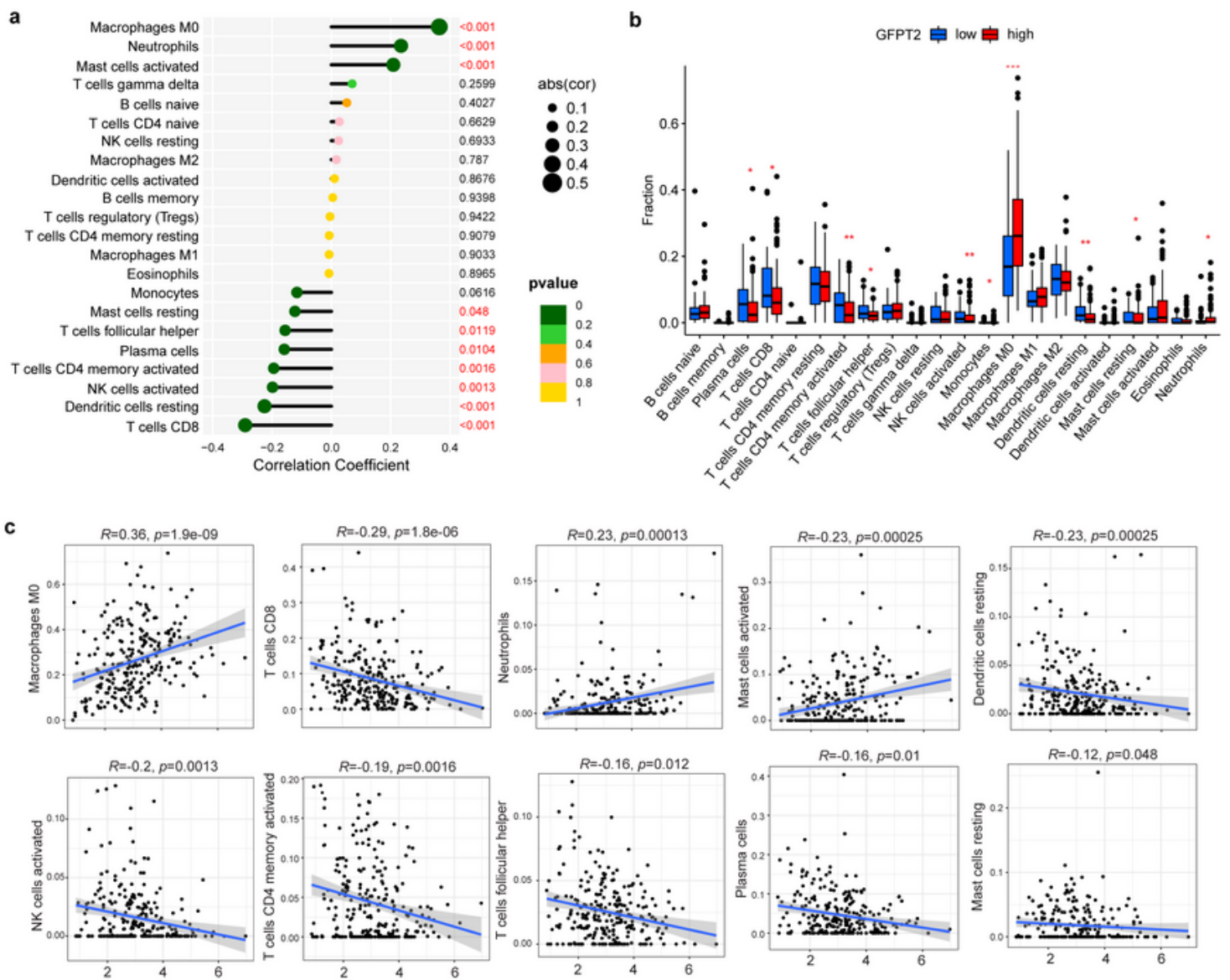

d
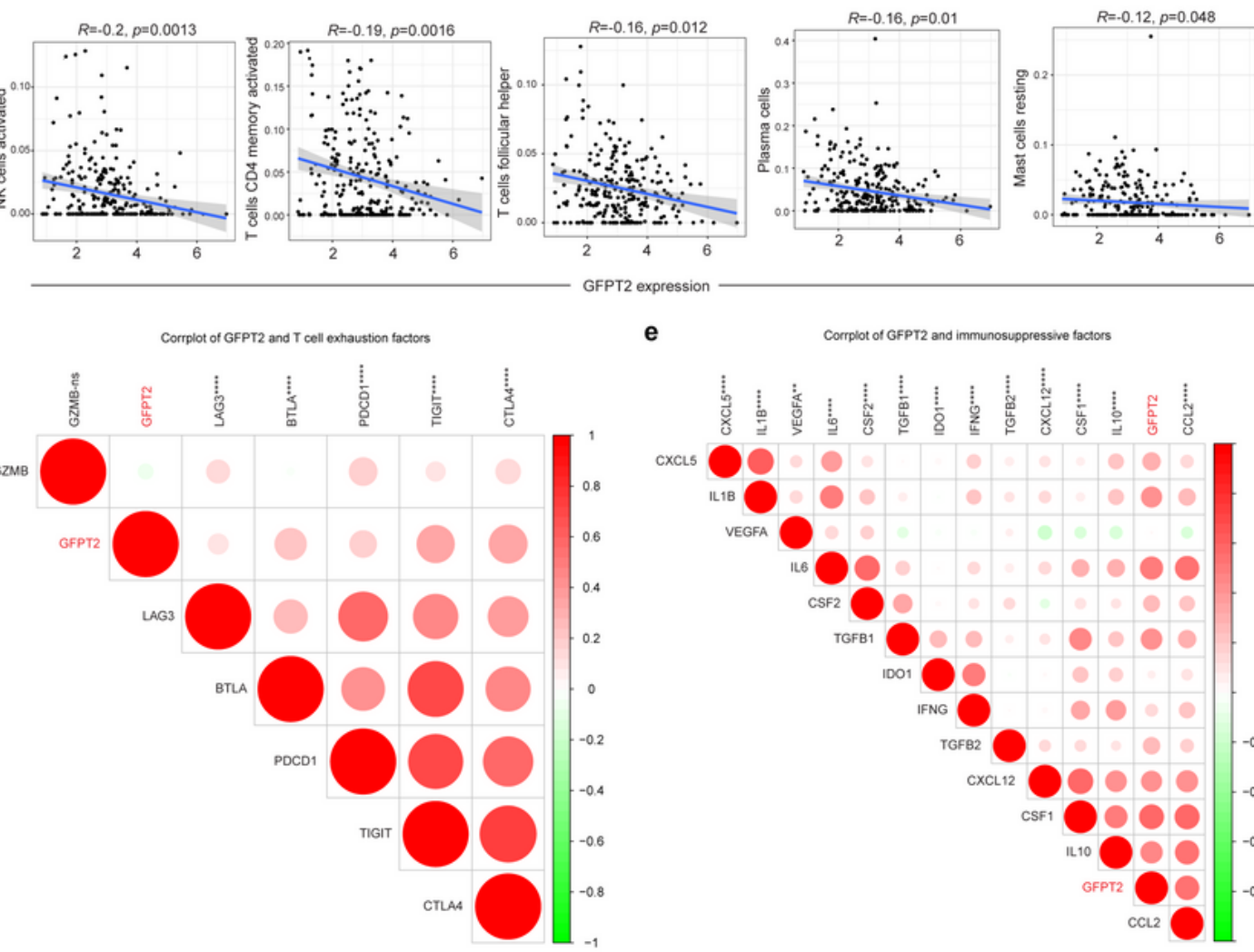

e

Corrplot of GFPT2 and immunosuppressive factors

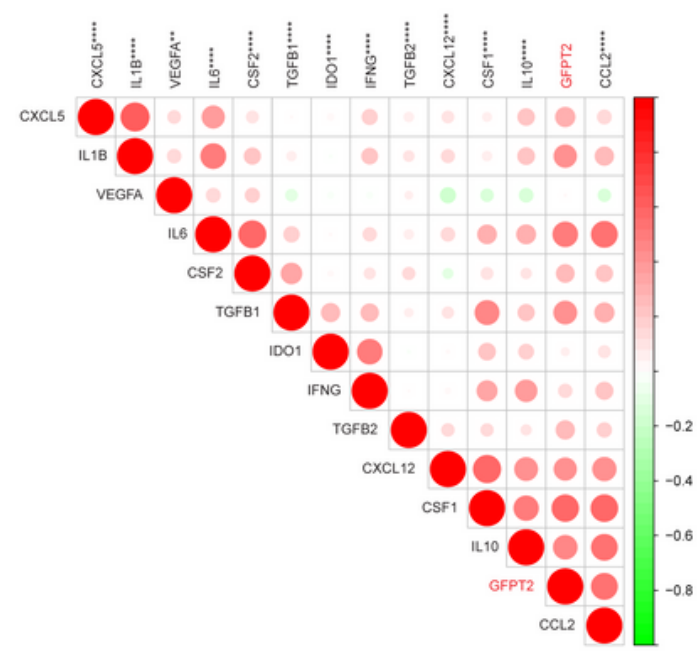

Figure 5

\section{Figure 5}

Association of GFPT2 levels with immune cell infiltration. a-c The correlation coefficients between GFPT2 expression and immune cells using the CIBERSORT method. $d$ Corrplot was used to perform the correlation between GFPT2 levels and T cell exhaustion factors. e Corrplot was used to perform the correlation between GFPT2 levels and immunosuppressive factors. 
GFPT2, Deforolimius

Cor $=0.496, p<0.001$

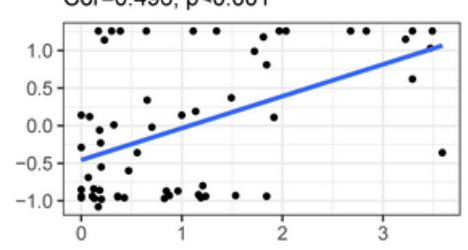

GFPT2, Motesanib

Cor $=0.381, p=0.004$

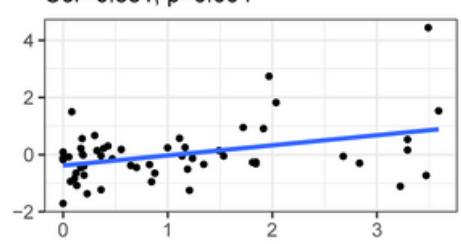

GFPT2, AZD-5363

Cor $=0.340, p=0.008$

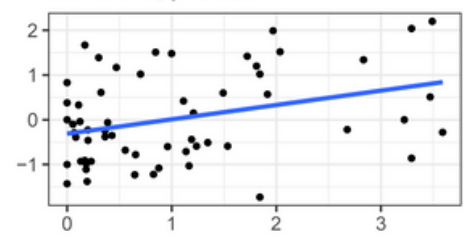

GFPT2, Rigosertib

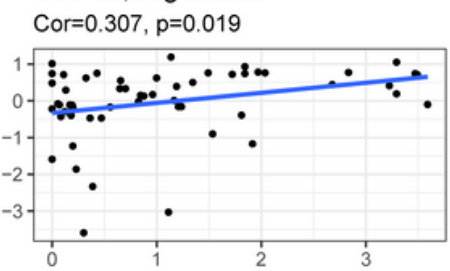

GFPT2, SGX-523

Cor $=0.464, p<0.001$

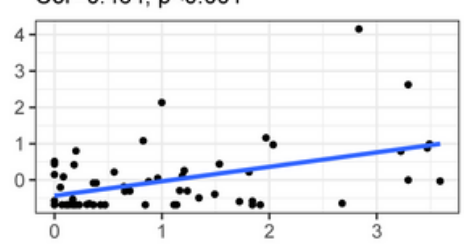

GFPT2, Staurosporine

Cor $=0.367, p=0.004$

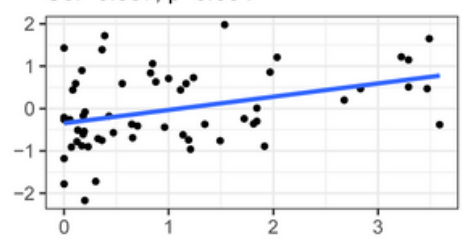

GFPT2, AS-703569

Cor $=0.354, p=0.011$

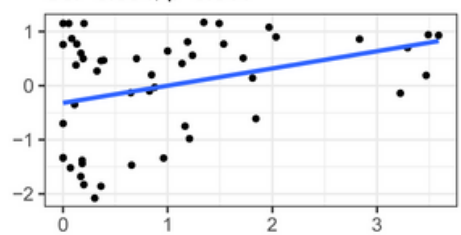

GFPT2, LY-294002

Cor $=0.304, p=0.020$

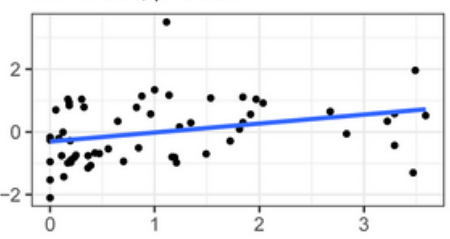

GFPT2, By-Product of CUDC-305

Cor $=-0.435, p<0.001$

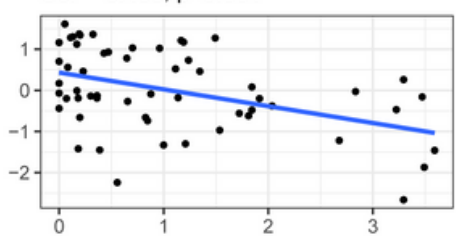

GFPT2, Itraconazole

Cor $=0.371, p=0.004$

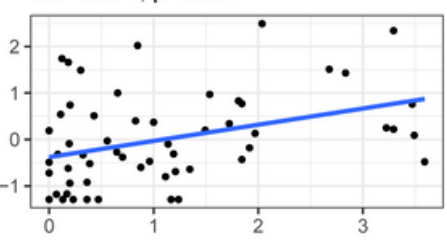

GFPT2, AT-9283

Cor $=0.320, p=0.014$

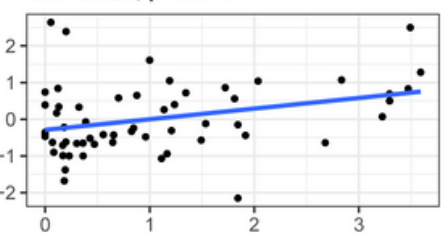

GFPT2, Rebimastat

Cor $=0.317, p=0.021$

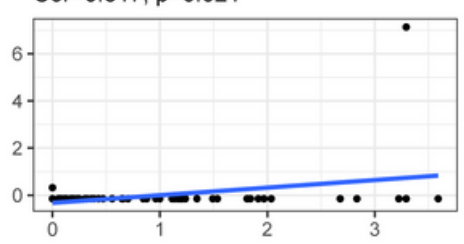

GFPT2, JNJ-38877605

Cor $=0.413, p=0.001$

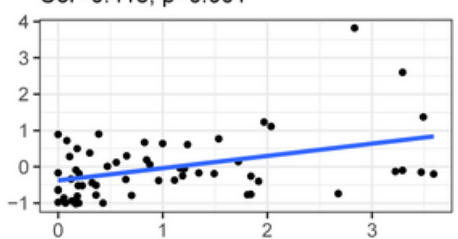

GFPT2, CCT-128930

Cor $=0.344, p=0.008$

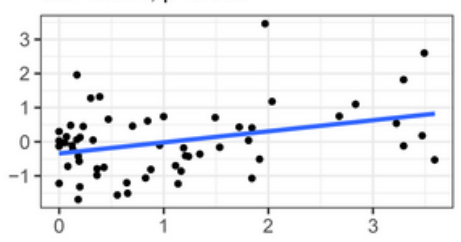

GFPT2, Silmitasertib Cor $=0.314, p=0.015$

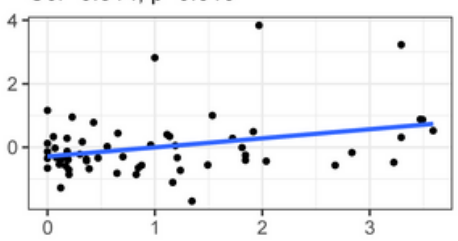

GFPT2, PF-04217903

Cor $=0.291, p=0.027$

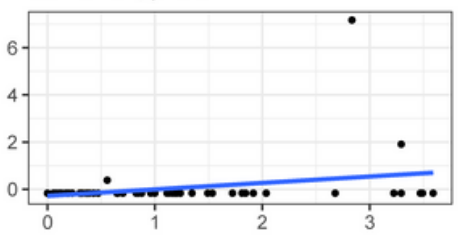

Figure 6

\section{Figure 6}

The relationship between GFPT2 expression levels and drug sensitivity. The correlation coefficient between GFPT2 expression and drug sensitivity from CellMiner dataset. 